\title{
FRONTERAS DE LA MINERÍA EN LA \\ PATAGONIA SUR ARGENTINA
}

\section{BORDERS OF MINING IN SOUTH \\ PATAGONIA ARGENTINA}

\author{
Alejandro Fabián Schweitzer*
}

\section{Introducción}

La Patagonia Sur se ubica en el extremo austral del continente americano, compartido entre Argentina y Chile. Conformada por las provincias de Santa Cruz y Tierra del Fuego, Antártida e Islas del Atlántico sur y departamentos del sur de la Provincia del Chubut, contaba en 2010 con 605.127 habitantes distribuidos en $313.897 \mathrm{~km}^{2}$, con una densidad de $1,93 \mathrm{hab} / \mathrm{km}^{2}$, es la región menos poblada y de menor densidad del territorio argentino ${ }^{1}$. Aunque en este artículo el análisis se centrará en el espacio argentino, también son parte de este espacio las regiones chilenas de Aysén del General Carlos Ibáñez del Campo y Magallanes y Antártica.
La incorporación del espacio patagónico al estado nacional se realizó desde sus inicios mediante la puesta en valor de sus recursos naturales. En primer lugar las pasturas, aguadas y sus nutrientes, como alimento para la expansión de la ganadería ovina y el circuito textil global; luego sus recursos minero-energéticos y la minería metalífera, así como la pesca de altura. Solo en los últimos años se expande el turismo, orientado también a la observación del paisaje natural.

La minería metalífera se inscribe en dinámicas globales de avance de las fronteras de mercantilización de la naturaleza, potenciada por reformas neoliberales realizadas en la década de 1990. El yacimiento Cerro Vanguardia en la Provincia de Santa

\footnotetext{
* Profesor de la Universidad Nacional de la Patagonia Austral (Santa Cruz/Argentina). Doutor en Geografía, Planejamento Territorial e Urbanismo. E-mail: alejandro.schweitzer@gmail.com

1. Ver Mapa 1. Patagonia Sur. Departamentos y localidades de más de 20.000 habitantes.
} 
Cruz, junto con Bajo La Alumbrera en la de San Juan, son caracterizadas como la primera generación de la minería metalífera en Argentina (BASUALDO, 2013).

De esta primera generación surgieron "aprendizajes" por parte de las empresas del sector, respecto a mecanismos de obtención de licencias sociales, pero también aparecen las primeras experiencias por parte de las sociedades y territorios en donde se realiza la actividad en término de impactos ambientales y sociales, por lo general negativos. En abril de 2003, fruto de apren- dizajes a partir de experiencias en otras provincias y países y meses de movilizaciones y un plebiscito realizado en la localidad de Esquel, en la Provincia del Chubut, que reflejó un 81\% de rechazo a la minería metalífera, el gobierno provincial promulgó la Ley 5001/03 (Digesto Jurídico Chubut), de prohibición de la minería a cielo abierto y el uso de cianuro. En diciembre de 2012 la Ley 853/11 (LEGISTDF 2019) prohibe la misma modalidad en la Provincia de Tierra del Fuego.

Mapa 1. Patagonia Sur. Departamentos y localidades de más de 20.000 habitantes.

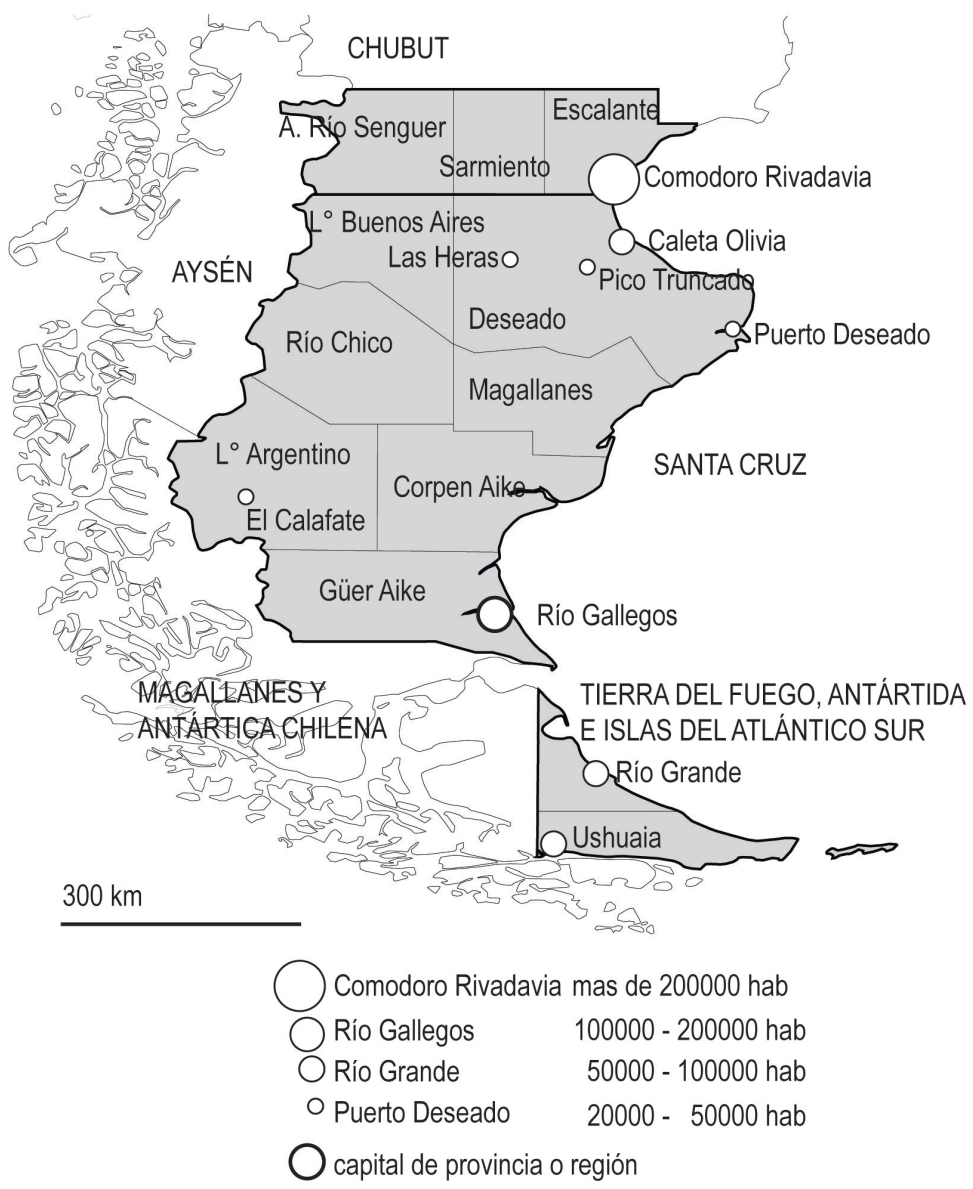

Elaboración: Schweitzer, A. Estimación de población 2019 en base a datos del INDEC (INDEC, 2019) 
La Provincia de Santa Cruz es la única en la Patagonia Sur que autoriza y promueve la actividad minera, aunque su realización generó resistencias. Entre 2003 y 2006 se conforman asambleas de vecinos contra la minería en las localidades de Los Antiguos, Perito Moreno, Las Heras y Gobernador Gregores, localidades cercanas a los yacimientos que estaban en etapa de exploración, así como en Río Gallegos, capital de la Provincia, en tanto sede de la empresa estatal provincial, de los organismos de control y las autoridades de aplicación en la actividad ${ }^{2}$. Entre estas organizaciones se realizan desde 2006 encuentros de intercambio de experiencias y organización de actividades. Desde 2004 las empresas por su parte despliegan estrategias con objeto de obtener la licencia social, en particular las agencias de desarrollo, en Puerto San Julián, Perito Moreno y Gobernador Gregores, localidades cercanas a los yacimientos y donde tienen sus oficinas.

En noviembre de 2009 el gobierno de Santa Cruz dicta la Ley 3105/09 creando el Area de Interés Especial Minero, que define un polígono en el cual se promueve la extracción de minerales metalíferos y la excluye del resto del territorio provincial, solo autorizando el funcionamiento del yacimiento de carbón de Río Turbio. Ac- tualmente se mantienen grupos en todas las localidades mencionadas, algunos activos mientras que otros programan actividades frente a posibles nuevos proyectos ${ }^{3}$.

En la Argentina, los resultados de censos de población y relevamientos de calidad de vida muestran que luego de un siglo en el caso del petróleo y el gas y de dos décadas en el de la minería metalífera, la renta de explotación de la naturaleza no fue aprovechada para mejorar la calidad de vida de la población y que en muchos casos redundaron en el empeoramiento de esta situación. El Índice de Calidad de Vida, elaborado en base a datos de censos nacionales de población y vivienda de 2001 y 2010, muestra un descenso generalizado en la calidad de vida de la población en la región. De los doce departamentos correspondientes a la Patagonia Sur, solamente uno muestra una leve mejora: Sarmiento, en la Provincia del Chubut, dedicado principalmente a la agricultura, mientras que dos en particular registran en fuerte deterioro: Deseado y Magallanes, en la Provincia de Santa Cruz, dedicados el primero a la extracción de petróleo y gas y el segundo a la minería metalífera (VELÁZQUEZ et al. 2014) ${ }^{4}$.

Existen diversos estudios sobre esta situación, referidos a la Patagonia y a la Pro-

2. En paralelo, en Comodoro Rivadavia (Provincia del Chubut), Caleta Olivia y Las Heras, otras agrupaciones iniciaban sus reclamos por contaminación de suelos y aguas superficiales, en particular por el uso del recurso hídrico por la extracción de hidrocarburos, que ocasiona también la falta de agua para casi ciento cincuenta mil habitantes de la cuenca petrolera del Golfo San Jorge.

3. Ya en esta década surgen en el centro y sur de la Provincia nuevas asambleas en oposición a la construcción de dos grandes represas sobre el río Santa Cruz. A diferencia de lo que sucede con la minería y los hidrocarburos en este caso se suman también las comunidades de pueblos originarios.

4. El Índice de Calidad de Vida es un indicador compuesto, elaborado a partir de dos dimensiones: socioeconómica, que comprende educación, salud y vivienda, y ambiental, que evalúa problemas ambientales y recursos recreativos (VELÁZQUEZ et al. 2014). 
vincia de Santa Cruz en particular, donde se señala que al deterioro de los indicadores oficiales y al Índice de Calidad de Vida se suman afectaciones ambientales, problemas de acceso al agua en calidad y cantidad, de contaminación de acuíferos, del aire y suelos y problemas sociales, como los derivados de la dualización de las sociedades entre aquellos que se encontraban vinculados a estas actividades dinámicas y los que no lo estaban y continuaban dependiendo de actividades menos dinámicas y tradicionales o del empleo público, problemas de acceso a la vivienda y al suelo urbanos, de inflación local, de trata de personas, prostitución y agravamiento de violencia de género (AGOSTO, 2008; PENGUE, 2008; SVAMPA, ANTONELLI, 2009; MARIN, 2009; MACHADO ARAOZ et al 2011; SCHWEITZER, 2011; GODOY; FASIOLI; VALIENTE; SCHWEITZER, 2013; SCHWEITZER; GODOY; FASIOLI, 2015; SCHWEITZER, 2016; SCHWEITZER; VALIENTE, 2018).

El objetivo de este artículo es exponer en primer lugar un marco para interpretar estos procesos, para lo cual se plantea como hipótesis que las dinámicas expansivas de la minería son parte de procesos globales de expansión de las fronteras de mercantilización de la naturaleza, en particular de recursos energéticos y materias primas. En un segundo momento se describe la evolución y el estado actual de la minería en la Provincia de Santa Cruz, que toma la forma de un proceso de producción del espacio y frontera de expansión del capital, centrándose en la megaminería metalífera. En tercer lugar se expone una suerte de balance de resultados y el estado actual de veinte años de presencia de la actividad en la provincia, así como de los conflictos y resistencias surgidas como oposición a la implantación de la actividad.

\section{Las fronteras de la mercantilización de la naturaleza en la Patagonia Sur}

Jason Moore sostiene que el desarrollo del capitalismo depende de sus capacidades de extenderse hacia espacios no mercantilizados, a la extensión de las relaciones de producción en vertical y horizontal (MOORE, 2013). La extensión vertical, hace referencia a espacios no mercantilizados de vida, como por ejemplo el trabajo no remunerado realizado por mujeres, esclavos o por naturaleza extra-humana, como los ríos, la energía solar o la geología planetaria. La extensión horizontal implica la expansión de la frontera de la mercantilización de la naturaleza para la apropiación de lo que el autor denomina los cuatro baratos -energía, alimentos, materias primas y trabajo-, creando así formas de naturaleza específicas al capitalismo. Para el autor, esto solo puede ser asegurado mediante "estrategias de apropiación fuera del circuito inmediato del capital" (MOORE, 2016, p. 163) y poco más adelante sugiere que "La frontera de productos es un viaje de ida. Las fronteras, una vez han sido apropiadas y comodificadas, dejan de ser fronteras" (MOORE, 2016, p. 163).

Este ensanchamiento continuo del espacio de apropiación de naturaleza barata, de expansión de fronteras se realiza mediante la internalización de espacios, así como la facilitación de la apropiación de trabajo, alimentos, energía y materias primas baratas para incorporarlos a los circuitos del capital, desde fuera hacia dentro. Tanto los momentos verticales -en el interior de los circuitos mediante la apropiación de trabajo no remunerado de la esfera de la reproducción hacia la de mercantilización, como los horizontales, por despliegues en espacios geográficos específicos, operan de manera unificada en el proceso de acumu- 
lación del capital.

El concepto de naturaleza barata se define así como un concepto histórico, se refiere a sociedades, tiempos y espacios concretos en los cuales un elemento natural se constituye como valor de uso final o como instrumento de trabajo de un circuito productivo, en los cuales el término "barato" puede referirse a los costos de producción o de extracción, a la facilitación del acceso al espacio de realización de esos procesos, en términos de propiedad o concesión. También se hace posible en la medida que el capital acceda a condiciones de producción particulares, que determinan las inversiones necesarias para su realización.

\subsection{El avance de las fronteras de mercantilización en la Patagonia sur}

La integración de la Patagonia sur a las dinámicas globales fue realizada en el marco de avances de las fronteras de mercantilización de la naturaleza. Desde el periodo posterior a las independencias nacionales se identifican en esta región tres fases.

La primera fase se extiende entre 1875 y 1907, donde en paralelo a la delimitación de la frontera binacional se estructura una región transfronteriza de derecho, piloteada por capitales originarios de Punta Arenas, asentada en su incorporación al circuito global de la producción textil motorizado desde el capitalismo central británico en el siglo XIX. Las condiciones para este avance de las fronteras fueron aseguradas por los estados nacionales, que aseguraron la integración de la región patagónica sur como espacio transfronterizo y por las empresas con base regional que construyó por si misma o junto a los estados argentino y chileno puertos, almacenes, caminos, frigoríficos, rutas, viviendas, infraestructura, equipamientos, seguridad y una serie de dispositivos legales y jurisdiccionales, para incorporar materia prima barata como suelo, pasturas y nutrientes así como la introducción de ganado ovino.

A fines de la década de 1880 las fronteras del circuito ovino-textil en el área chilena alcanzaron los límites impuestos por las condiciones naturales y traspasaron la frontera política hacia los territorios nacionales de la Patagonia sur argentina. Este carácter transfronterizo fue refrendado con la firma del Tratado de Paz, Amistad, Comercio y Navegación entre la República de Chile y la Confederación Argentina, que promovía la libre circulación de personas, capitales y mercancías. Firmado en 1856 $\mathrm{y}$ vigente con escasas interrupciones hasta 1912, el objetivo del tratado apuntaba a potenciar las interacciones, que separaban las funciones de frontera comercial -suspendida por el tratado- de sus funciones políticas, dependientes del avance en los tratados de fijación de límites y la demarcación de las fronteras. El circuito textil fue complementado por el cárnico con la implantación de los frigoríficos desde inicios del siglo XX.

La producción del espacio y la configuración del territorio en el primer periodo se realizó para atender las necesidades del modelo primario-exportador asentado en el circuito textil, dando lugar a un espacio regional de acumulación de capital transnacional, integrado de manera subordinada a la economía-mundo hegemonizada por Gran Bretaña (SCHWEITZER, 2016).

En 1907 comienza el segundo periodo. Contenida integralmente en la Plataforma Patagónica, elevada hacia el Oeste en la cordillera de los Andes y que se extiende sobre la plataforma continental, esta segunda frontera sera minera-energética, motorizada por la 
extracción de petróleo y gas en primer lugar en la cuenca San Jorge y desde la década de 1960 sobre la Austral-Magallánica. Esta fase se extiende hasta la década de 1990.

El periodo se inicia con el descubrimiento de petróleo en Comodoro Rivadavia, en el Territorio Nacional del Chubut. La implantación del circuito de los hidrocarburos fue objeto de políticas del estado nacional argentino, que en 1922 fundó la empresa Yacimientos Petrolíferos Fiscales (YPF). Esta empresa hasta fines de la década de 1950 no solamente competía con capitales privados nacionales e internacionales y se reservaba la comercialización de combustibles, controlando así el mercado nacional en su totalidad. En 1946 comienza la extracción de carbón en Río Turbio, inicialmente como una división específica al interior de YPF, luego escindida en Yacimientos Carboníferos Fiscales ${ }^{5}$. En la década de 1960 se inicia la extracción de gas y petróleo en la cuenca Austral, compartida con Chile. Hasta fines de la década de 1970 la empresa aportó a la ocupación y poblamiento de la región, con políticas sectoriales de vivienda, equipamiento e infraestructura pública en asentamientos que llegaron a contar con niveles relativamente altos de calidad de vida. Este periodo, donde el sector minero-energético orientado a proveer el mercado interno organiza la economía y la sociedad locales, se extendió hasta la década de 1990 con la culminación de la privatización de YPF
(SCHWEITZER, 2012).

A mediados de la década de 1990 se inicia el tercer período, que se extiende hasta la actualidad. En 1992 la Argentina comienza a ser exportadora de hidrocarburos, principalmente en las cuencas del Golfo San Jorge y Austral y desde la década de 2000 se desarrollan la pesca, el turismo y la minería metalífera. Se configura así un espacio más diversificado en los "productos" extraídos y de nuevos avances de las fronteras de mercantilización de la naturalezs, que justifica su diferenciación respecto a los anteriores. Esta fase fue potenciada por el "consenso de los commodities" y la penetración de grandes capitales transnacionales (SVAMPA, 2012). En los últimos años se va expandiendo la extracción de hidrocarburos no convencionales por fractura hidráulica (fracking) como nuevo método para extracción de hidrocarburos.

La actividad minero-energética lleva ya más de un siglo y aun hoy sigue siendo uno de los principales productos de exportación y fuente de ingresos de los dos estados provinciales. Desde la década de 1990 de la mano de las políticas privatistas derivadas de la consolidación de regímenes neoliberales, esta frontera será repotenciada avanzando sobre la plataforma continental argentina hasta las islas Malvinas. Coincidirá también con la expansión de la frontera de la minería metalífera.

5. En 1955 la Ley 14408 dispone la creación de las provincias del Cubut y de la Patagonia, integrada por los antes territorios nacionales de Santa Cruz y Tierra del Fuego. Esta última fue disuelta al año siguiente, resultando en la creación de la Provincia de Santa Cruz y la restitución del Territorio Nacional de Tierra del Fuego. Esta última se constituyó finalmente como Provincia recién en 1990. 


\subsection{El "peso" de las fronteras de mercantilización y su integración en dinámicas de acumulación globales}

Detrás de la región pampeana, la Patagonia sur es la segunda respecto al valor de las exportaciones, centradas principalmente en productos primarios como gas, petróleo, minerales metaliferos y pesca (Ver Tabla 1). La ganadería, actividad tradicional en la región, tiene cada vez menor peso en este rubro.

En relación al peso de las exportaciones respecto al producto geográfico bruto, las provincias de Chubut y Santa Cruz son muy dependientes del mercado externo ${ }^{6}$. Ocupan respectivamente el $4^{\circ}$ y $5^{\circ}$ lugar en exportaciones, solo superadas por las provincias de Buenos Aires, Santa Fe y Córdoba (INDEC 2017).

La Provincia de Tierra del Fuego en cambio, de acuerdo a la misma fuente, ten- dría escaso peso en las exportaciones. Esta información queda relativizada dado que en la Argentina la extracción offshore, por más que se realice dentro de las 12 millas de jurisdicción marítima de las provincias, se contabiliza como proveniente de la Plataforma marítima y no de las provincias, por lo que no se computan como exportaciones de origen provincial ni entran en los cálculos de PGB. En la composición de exportaciones totales de Tierra del Fuego, con la salvedad del párrafo anterior, se destacan los pescados y mariscos sin elaborar y elaborados con el 55,4\%, mientras que el gas y el petróleo engloban el 16,05\% (Secretaría de Política Económica, 2018). En la Provincia se localizan importantes capitales trasnacionales del sector hidrocarburos, en particular las empresas TOTAL e YPF.

Tabla 1. Origen provincial de las exportaciones Año 2017

\begin{tabular}{|c|c|c|c|c|c|}
\hline \multirow{3}{*}{ Provincia } & \multirow{2}{*}{$\begin{array}{l}\text { Valor } \\
\text { total de expor- } \\
\text { ta-ciones }\end{array}$} & \multirow{2}{*}{$\begin{array}{c}\text { Aporte de exporta- } \\
\text { ciones sobre total } \\
\text { nacional }\end{array}$} & \multicolumn{3}{|c|}{ Principales rubros exportados } \\
\hline & & & \multirow{2}{*}{$\begin{array}{l}\text { Primario (in- } \\
\text { cluye pesca) }\end{array}$} & \multirow{2}{*}{$\begin{array}{l}\text { Productos de ori- } \\
\text { gen industrial } \\
\text { (incluye minería) }\end{array}$} & \multirow{2}{*}{$\begin{array}{c}\text { Combustibles } \\
\text { y energía }\end{array}$} \\
\hline & $\begin{array}{c}\text { Millones de } \\
\text { dólares }\end{array}$ & $\%$ & & & \\
\hline Chubut & 2196 & 3,7 & 820 & 761 & 445 \\
\hline Santa Cruz & 2090 & 3,6 & 593 & 1366 & 119 \\
\hline Tierra del Fuego & 158 & 0,3 & 70 & 43 & 24 \\
\hline
\end{tabular}

Elaboración propia. Fuente: INDEC. Origen provincial de las exportaciones Año 2017. Informes Técnicos vol. $2 n^{\circ} 40$ Comercio exterior vol. 2 n 5 . Buenos Aires, 2018.

También para 2017, en el caso del Chubut, estas exportaciones se dividen en $35,1 \%$ pescados y mariscos sin elaborar, 34,39 aluminio y 21,08 petróleo crudo, mientras que en Santa Cruz, el 71,1\% de sus exportaciones consistió en 2017 en oro, plata y otros minerales metalíferos, 22,3\% pescado y mariscos y tan solo 5,6\% en petróleo.

Chubut y Santa Cruz incorporan el gas al mercado nacional por el gasoducto San Martín, por lo cual a su importante rol en

6. Este dato se trata de una estimación ya que el PGB no se calcula desde 2005. 
las exportaciones debe sumarse su aporte al mercado interno. Tierra del Fuego exportó hasta 2011 gas licuado hacia Chile, con un valor en ese año de 201 millones de dólares, equivalentes al 45\% del valor total de las exportaciones de la provincia ese año. La caída en este rubro se debió al cambio de la matriz energética de Chile y redespliegue de la frontera de los hidrocarburos en ese país, principalmente por medio de la fractura hidráulica. Los productos de la minería metalífera de Santa Cruz es exportada casi en su totalidad a Suiza, donde se realizan procesos de refinado.

El peso de las fronteras de mercantilización de los cuatro baratos se refleja en los principales productos exportados, que se exponen en la Tabla 2. El "cuarto barato" es el trabajo humano, que no se contabiliza aunque se encuentra cristalizado en las tareas de extracción y el escaso procesamiento de gran parte de esos productos.

Tabla 2. Alimentos, energía y materias. Principales exportaciones de la Patagonia Sur

\begin{tabular}{|l|l|l|l|}
\hline & Millones de US\$ & Tipo de materia & Provincia \\
\hline Aceites crudos de petróleo & 592,2 & Energía & Chubut \\
\hline Oro & 438,2 & Mat. Primas & Santa Cruz \\
\hline Petróleo crudo & 428,1 & Energía & Santa Cruz \\
\hline Plata & 373,8 & Mat. Primas & Santa Cruz \\
\hline Aluminio sin alear & 328,1 & Mat. Primas & Chubut \\
\hline Camarones y langostinos & 260,6 & Alimentos & Chubut \\
\hline Langostinos & 228 & Alimentos & Santa Cruz \\
\hline Camarones y langostinos congelados & 188,5 & Alimentos & Chubut \\
\hline Aleaciones de aluminio & 179,1 & Mat. Primas & Chubut \\
\hline
\end{tabular}

Elaboración propia. Fuentes: Secretaría de Política Económica, 2018. informes provinciales de Chubut, Santa Cruz y Tierra del Fuego, con datos de 2017.

\section{La expansión reciente de la minería metalífera en la Patagonia Sur}

Existen numerosos estudios que coinciden en identificar, durante la década de 1990 y hasta mediados de la década pasada, una carrera por mayores niveles de mercantilización del suelo y subsuelo y la extracción de minerales metalíferos en el escenario global ya mencionado del Consenso de los Commodities y más recientemente, de la mano de nuevas inversiones chinas, del Consenso de Beijing (RODRIGUEZ PARDO, 2009; SVAMPA; SLIPAK, 2015). En el caso de la Patagonia, se trata de energía, en tanto permanencia de la frontera de los hidrocarburos y el carbón, a los que se agrega desde
1997 la minería metalífera centrada en el oro y la plata. La expansión de la frontera minera en la región en la década de 1990, fue posible por la existencia de estos recursos y por el desenvolvimiento de procesos iniciados dos décadas antes, con los gobiernos militares con la imposición de gobiernos neoliberales y su permanencia en periodos de gobierno constitucional por la ocupación de los bancos centrales y ministerios de economía por funcionarios que obedecían a estas orientaciones, cuando no directamente ex-gerentes de grandes empresas. Estos sectores, siguiendo recomendaciones de organismos internacionales como el Banco Mundial, el Fondo Monetario Internacional y la Organización Mundial de Co- 
mercio forzaron hacia fines de la década de 1980 y más fuertemente en la siguiente, a la privatización de activos nacionales, lo que incluyó la concesión del subsuelo.

En la Argentina los datos más recientes del Producto Geográfico Bruto por provincia son de 2005, apenas iniciada la actividad minera, por lo cual no pueden ser tomados en cuenta. Las fuentes oficiales de generación de empleo por la actividad engloban en la misma a la minería metalífera, de carbón, la extracción de materiales de canteras y la extracción de hidrocarburos, por lo cual tampoco es fácil inferir el empleo concreto de la minería metalífera. En su conjunto, en la Provincia de Santa Cruz se informa que en 2017 generaban 17000 puestos entre el sector privado y el publico, solo superada por el sector servicios con 22000 puestos (Secretaría de Política Económica, 2018). De esos 17000 puestos, mas de 2000 corresponden a la extracción de carbón en Río Turbio, a cargo de Yacimientos Carboníferos Río Turbio, una empresa pública, del estado nacional argentino. Por su porte, el Código de Minería es nacional, actualizado en 1997 y agrupa en los de primera categoría a los metales, los combustibles sólidos y las piedras preciosas (Ley 1919/97 Infoleg) por lo cual tampoco es fácil diferenciar el aporte concreto de la minería metalifera a la economía o al desarrollo regionales.

En la Provincia de Santa Cruz la exportación de oro constituía en 2016 más del $50 \%$ del valor total de sus exportaciones, superando al petróleo y el gas (Secretaría de Política Económica, 2018). Estos tres productos, junto al turismo y la pesca, conforman practicamente la totalidad de actividades realizadas por el sector privado $\mathrm{y}$ parte del público por medio de su principal empresa provincia, FOMICRUZ Sociedad del Estado, que participa de la explotación minera en asociación con grandes capitales transnacionales. También comienza recientemente a incursionar en hidrocarburos, en este caso también con capitales nacionales.

A efectos expositivos, se presentan en primer lugar las modificaciones legales, impositivas y jurisdiccionales, como parte del conjunto de dispositivos por los cuales una región donde hasta la década de 1990, con excepción del carbón, apenas se realizaba alguna extracción artesanal de mineral de oro, se transformó en una de las regiones más activas, en particular en el caso argentino. En segundo lugar se presenta el estado actual de la minería en la región.

\subsection{Dispositivos de apoyo a la expansión de la frontera de la minería metalífera}

Entre la primera mitad de la década de 1990 y la actualidad se registra una ola de modificaciones en la gestión de la actividad minera a lo largo de América Latina. Estas modificaciones han sido propuestas por grandes capitales transnacionales y organismos multilaterales de crédito, se ajustaron dispositivos de diversa índole, entre otros, los marcos legales, los regímenes impositivos y las medidas de promoción, a escala nacional y en la Argentina, también en provincias y municipios.

En el plano legal, en 1997, por Ley 1919/97 se reformó el antiguo Código de Inversiones Mineras, que ya tenía casi un siglo de existencia. El nuevo código actualiza el marco regulatorio de la actividad. Se dispone así la libre disponibilidad de los recursos por parte de las empresas, estabilidad fiscal y un régimen impositivo especial que incluye mayores deducciones de gastos de exploración, beneficios para amortizar inversiones de capital, devolución de impuestos, no pago 
de derechos de importación de maquinarias y repuestos, entre otros. Con este marco regulatorio se establece además un tope máximo del 3\% de regalías sobre el valor en boca de mina que podrían cobrar las provincias y en particular la prohibición de participación en la actividad extractiva por parte de las provincias, salvo mediante asociación con capitales privados. Por otro lado y con carácter más general, desde 1985 y con sucesivas prórrogas hasta 2016 estuvo en vigencia el reembolso adicional a exportaciones por puertos de la Patagonia establecido por ley 23018/83 y prorrogado por Decreto 2229/15, mediante el cual se disponía una devolución de entre el 8\% en el puerto de San Antonio Este, en la Provincia de Río Negro, Patagonia norte y el 13\% en el puerto de Ushuaia, en el extremo sur de la Patagonia.

En el plano jurisdiccional, en 1997 se firman el Tratado sobre Integración y Complementación Minera y el Protocolo Complementario del Tratado de Integración y Complementación Minera entre Chile y Argentina, que fue ratificado y aprobado en 2000 por Ley 25243/00 (Infoleg). Este tratado determina una franja a lo largo de la frontera binacional destinada al fomento de la actividad minera, y suspende las restricciones vigentes en ambas naciones referidas al acceso a la propiedad, derechos mineros y al ejercicio de la actividad en general. Permite también la libre circulación de maquinarias por la frontera, la consideración como "nacional" a empresas de los dos países. Este acuerdo en los hechos no dio lugar a mayores avances en la explotación, con excepción del yacimiento de Pascua Lama, en la Provincia de San Juan, región de Cuyo en Argentina y del Atacama en Chile (SCHWEITZER; GODOY; RAUQUE, 2011; GIARRACCA; TEUBAL, 2013). El acuerdo se impulsó nuevamente en el año 2015, sin embargo quedó trunco debido a los cambios de gobierno, y finalmente en octubre de 2017 se retomó la negociación de su actualización y la prolongación de su vigencia.

En las dos primeras décadas del presente siglo se pusieron en práctica algunas restricciones. En el 2003, por Ley 5001/03 se prohibió la minería en la Provincia del Chubut (Digesto provincial 2003) y en 2012 en Tierra del Fuego mediante la ley 853/11. Por el contrario en la provincia de Santa Cruz el estado apuesta con fuerza en al impulso de la actividad. En 2009 la cámara de diputados local promulga la ley 3105/09 de creación del “Area de Interés Especial Minero para la realización de actividades mineras" (MINPRO 2009), que establece un área de promoción especial de la actividad. En 2010 se aprueba en Argentina se aprueba por Ley 26639/10 el Régimen de presupuestos mínimos para la preservación de los glaciares y del ambiente periglacial (INFOLEG, 2010). Dicha legislación entró en contradicción con algunas empresas mineras ya que demandó la realización del inventario nacional de glaciares y prohibió la actividad minera en dichas zonas.

Con el cambio del gobierno nacional en 2015 y posteriormente en 2016, se disminuyeron las retenciones a las exportaciones de minerales de plata y los derechos de importación, así como algunos impuestos nacionales, en particular los Ingresos Brutos, que fueron eliminados bajando sensiblemente el aporte de la actividad a las rentas nacionales. En el acuerdo denominado "Pacto fiscal" realizado entre el gobierno nacional y las provincias en 2017 se incluian estas medidas y algunas políticas concretas. En particular, el acuerdo Federal Minero de 2017, realizado por el Consejo Federal de Minería (COFEMIN), buscó sintetizar y homogeneizar los instrumentos provinciales 
vigentes, e incluyó cambios en la forma de liquidar regalías que se mantenían en un $3 \%$ y se liquidarían sobre la facturación, por lo que habría mayores posibilidades de control de la extracción (TAPIA; 2017). El acuerdo federal hasta el momento no logró renovar el impulso de la actividad minera, que en algunos casos, de acuerdo a entrevistas realizadas con la empresa, se encuentra realizando planes de cierre, como es el caso de Mina Martha y Cerro Vanguardia, en la Provincia de Santa Cruz, o Bajo la Alumbrera en la Provincia de Catamarca.

La Provincia del Chubut no fue firmante de este acuerdo debido a la prohibición de la minería de uranio, mientras que la provincia de Santa Cruz profundizó sus medidas en favor de la actividad. En 2016 Santa Cruz se creó el Programa de Desarrollo de Proveedores (PRODEPRO), mientras que el Ministerio de Producción aprobaba un subsidio para las tasas de interés en créditos de las empresas mineras y establecía asistencias técnicas. Se continuó así con el fortalecimiento de la empresa Fomento Minero de Santa Cruz (FOMICRUZ SE), mediante la cual el estado provincial se dedica a la prospección, exploración y explotación minera y petrolera.

En 2013 se aprobó la Ley 351/13 de fortalecimiento financiero provincial (diario El nuevo día, 14/06/2013) que establecía entre otros el impuesto al derecho real de propiedad inmobiliaria minera, que imponía el pago del 1\% sobre el valor de las reservas en la compra venta de propiedades con yacimientos. Este impuesto fue derogado en 2015. En 2016 se crea el Fondo fiduciario Unión por la Responsabilidad Social Empresarial (UNIRSE) que prevé aportes voluntarios de empresas mineras (Cerro Negro, Cerro Vanguardia y San José entre otras) situadas en la provincia de Santa Cruz, para destinarlos a inversión social, obras y proyectos productivos.

Estas medidas implementadas por los estados son parte de un plan general implícito en documentos oficiales de organismos de planificación territorial y obras públicas nacionales y subcontinentales. Las dos ediciones del Plan Estratégico Territorial Argentina 2016 (2006/2007 y 2008/2009), así como sus actualizaciones hasta 2018 y varios planes provinciales, promovían bancos de proyectos y listados de obras públicas en los cuales se incluyeron obras al servicio de la minería, en particular la extensión de gasoductos y tendidos de energía eléctrica (SECRETARÍA DE PLANIFICACIÓN TERRITORIAL Y COORDINACIÓN DE OBRA PÚBLICA, 2018). Por otro lado, en el marco de la Iniciativa para la Integración de las Infraestructuras Sudamericanas (IIRSA) y el COSIPLAN, en el 2010 fueron definidos y aprobados para su concreción 10 ejes que articulan más de 500 proyectos, algunos de ellos megaproyectos, de transporte, energía y comunicaciones. Uno de estos ejes, el del Sur, atraviesa el sur de la Provincia del Chubut y norte de Santa Cruz, definiendo un corredor y priorizando pasos de frontera y puertos de salida de exportaciones que contemplan a la minería como una de las actividades que sería beneficiada por su construcción.

Desde 2004 las empresas por su parte despliegan estrategias con objeto de obtener la licencia social, en particular las agencias de desarrollo, en Puerto San Julián, Perito Moreno y Gobernador Gregores, localidades cercanas a los yacimientos y donde tienen sus oficinas. Entre 2004 y 2006 se crean agencias de desarrollo en Puerto San Julián, Perito Moreno y Gobernador Gregores, que siguen el modelo de las creadas en 1999 y 2003, en Pico Truncado y Caleta Olivia, relacionadas con la extracción de hidrocarburos (OBSERVADOR CENTRAL, 2014). Son organismos mixtos, con aportes de las em- 
presas mineras y que con variantes suman en sus directorios a gobiernos municipales, cámaras de comercio y de proveedores de servicios a las mineras, sociedades rurales, sindicatos del sector y universidades. Asimismo, estas empresas adoptan el discurso de la minería "sustentable" y en casos de espacios poco poblados como el de la $\mathrm{Pa}$ tagonia, se asumen como instrumento del progreso, el desarrollo y el poblamiento de espacios vacíos (SCHWEITZER, 2013).

\subsection{El estado actual de la minería en la Patagonia sur}

La minería avanza desde la década de 1990 mediante la participación de grandes inversiones de capitales transnacionales y con momentos de alzas y bajas, con la extracción de recursos en el marco de la financiarización de la economía mundial y la provisión de recursos mineros metalíferos en función de la demanda de China o la especulación con los precios de los commodities.

En las dinámicas de esta actividad algunos autores diferencian dos etapas, la primera entre 1990 y 2003, centrada en reformas a los marcos regulatorios, ya expuestos en el apartado anterior, y el inicio de la exploración y la habilitación de los dos primeros grandes emprendimientos, Cerro Vanguardia en la Provincia de Santa Cruz es uno de estos. La segunda etapa se extiende hasta el 2010 y se caracteriza por el alza de los precios internacionales desde 2003, la proliferación de la actividad de exploración, y la apertura de nuevas explotaciones (BASUALD0, 2013). A partir del 2011 se observan procesos como, el descenso de los precios internacionales, el comienzo del cierre de algunas explotaciones por haber concluido la extracción de los yacimientos, demora en la entrada de operación de nuevas inversiones, así como el retiro de empresas de la región por problemas de costos. Se trataría posiblemente de una de retracción, si no fuera por la continuidad de la presión de estos grandes capitales para la apertura de nuevas áreas de extracción, es decir, el acceso a nuevas materias primas baratas.

En términos estadísticos, entre 2003 y 2016, en todas las provincias de la Patagonia argentina hubo un incremento en la cantidad de establecimientos en todos los tipos de extracción con excepción de la Provincia de Río Negro, que experimentó un pequeño descenso. Las provincias con mayor crecimiento fueron Neuquén y Tierra del Fuego, aunque en esta última poco más de la mitad estaba en producción en 2016 (INDEC, 2017). El incremento de la actividad en las provincias con prohibición, como el caso de Tierra del Fuego, puede deberse a otros minerales no metalíferos, de canteras o carbón. En la Tabla 3 se señalan los yacimientos de minerales con algún tipo de actividad, las empresas a cargo y el tipo de mineral extraído, en la Provincia de Santa Cruz

En esta provincia las empresas Fomento Minero de Santa Cruz (FOMICRUZ Sociedad del Estado), Yacimientos Carboníferos de Río Turbio (YCRT) y CIMINAS son las únicas empresas mineras de capitales nacionales. En el caso de FOMICRUZ S.E participa de la actividad en etapas de prospección y de exploración y está asociada en Cerro Vanguardia SA con la empresa anglo-sudafricana Anglo Gold Ashanti, que mantiene el 92,75\% del capital desde 1998.

En el trabajo de Elsa Bruzzone (2012) acerca de la situación de la minería en Argentina, se registra una concentración de empresas, con ventas, fusiones y concesiones que caducan y pasan a manos de otras. La novedad más importante en términos de capitales es la irrupción de empresas chi- 
nas. En el caso de otros minerales, no metalíferos, la minería de carbón se realiza por galería, y a cargo del estado en la empresa estatal Yacimientos Río Turbio.

En la tabla, $n^{\circ} 3$ se exponen datos de los emprendimientos mineros en la Provin- cia de Santa Cruz, actualizados a 2016. El mapa $n^{\circ} 2$ permite por su parte visualizar los espacios de extracción de minerales metaliferos y energéticos en las provincias de Santa Cruz y Tierra del Fuego.

Tabla 3. Empresas mineras en la Provincia de Santa Cruz

\begin{tabular}{|c|c|c|c|c|c|}
\hline Yacimiento & \multicolumn{2}{|c|}{ Titular del derecho y exploradora/explotadora } & $\begin{array}{l}\text { Fase del } \\
\text { proyecto }\end{array}$ & Mineral & $\begin{array}{l}\text { Campamento } \\
\text { / Localidad } \\
\text { cercana }\end{array}$ \\
\hline Río Turbio & \multicolumn{2}{|l|}{ YCRT } & $\begin{array}{l}\text { Explotación } \\
\text { (en declive) }\end{array}$ & Carbón & $\begin{array}{l}\text { Río Turbio y } 28 \\
\text { de noviembre }\end{array}$ \\
\hline La Josefina & \multicolumn{2}{|l|}{ FOMICRUZ S.E } & Exploración & Oro y plata & $\begin{array}{l}\text { Gobernador } \\
\text { Gregores }\end{array}$ \\
\hline Don Nicolás & \multicolumn{2}{|l|}{ Don Nicolás S.A } & Explotación & Oro y plata & Tres Cerros \\
\hline Cerro Moro & Estelar ressources - Yamana Gold & Explotación & Oro y plata & Puerto Deseado & \\
\hline Bahía Laura & FOMICRUZ S.E & Exploración & Oro y plata & Puerto Deseado & \\
\hline $\begin{array}{l}\text { Cerro } \\
\text { Vanguardia }\end{array}$ & $\begin{array}{l}\text { Anglogold Ashanti + FOMICRUZ } \\
\text { S.E }\end{array}$ & Explotación & Oro y plata & $\begin{array}{l}\text { Puerto San } \\
\text { Julián }\end{array}$ & \\
\hline $\begin{array}{l}\text { Manantial } \\
\text { Espejo }\end{array}$ & $\begin{array}{l}\text { Minera Triton - Pan American } \\
\text { Silver }\end{array}$ & Explotación & Oro y plata & $\begin{array}{l}\text { Gobernador } \\
\text { Gregores }\end{array}$ & \\
\hline San José & Minera Santa Cruz - Hotschild & Explotación & Oro y plata & Perito Moreno & \\
\hline Cerro Negro & Oroplata - Goldcorp & Explotación & Oro y plata & Perito Moreno & \\
\hline $\begin{array}{l}\text { La Paloma - Lo- } \\
\text { mada de Leiva }\end{array}$ & Patagonia Gold & Explotación & Oro y plata & Perito Moreno & \\
\hline El tranquilo & Patagonia Gold & Exploración & Oro y plata & $\begin{array}{l}\text { Gobernador } \\
\text { Gregores }\end{array}$ & \\
\hline La Manchuria & Patagonia Gold & Exploración & Oro y plata & $\begin{array}{l}\text { Gobernador } \\
\text { Gregores }\end{array}$ & \\
\hline Mina Martha & Coeur Mining & $\begin{array}{l}\text { Cierre de } \\
\text { mina }\end{array}$ & Oro y plata & $\begin{array}{l}\text { Gobernador } \\
\text { Gregores }\end{array}$ & \\
\hline Joaquín & Coeur Mining & Exploración & Pllata & $\begin{array}{l}\text { Gobernador } \\
\text { Gregores }\end{array}$ & \\
\hline $\begin{array}{l}\text { El Dorado - } \\
\text { Monserrat }\end{array}$ & 5R - Samco Gold & Exploración & Oro y plata & Jaramillo & \\
\hline Las Calandrias & Mariana Ressources & Exploración & Oro y plata & $\begin{array}{l}\text { Puerto San } \\
\text { Julián }\end{array}$ & \\
\hline Pingüino & SCRN - Argentex & Exploración & Oro y plata & Puerto Deseado & \\
\hline
\end{tabular}

Elaboración propia con datos de Plano de proyectos mineros 2016. Secretaria de Estado de Minería, Ministerio de Producción de Santa Cruz. 
Mapa 2. Actividades extractivas minero-energéticas y minero-metalíferas en la Patagonia Sur

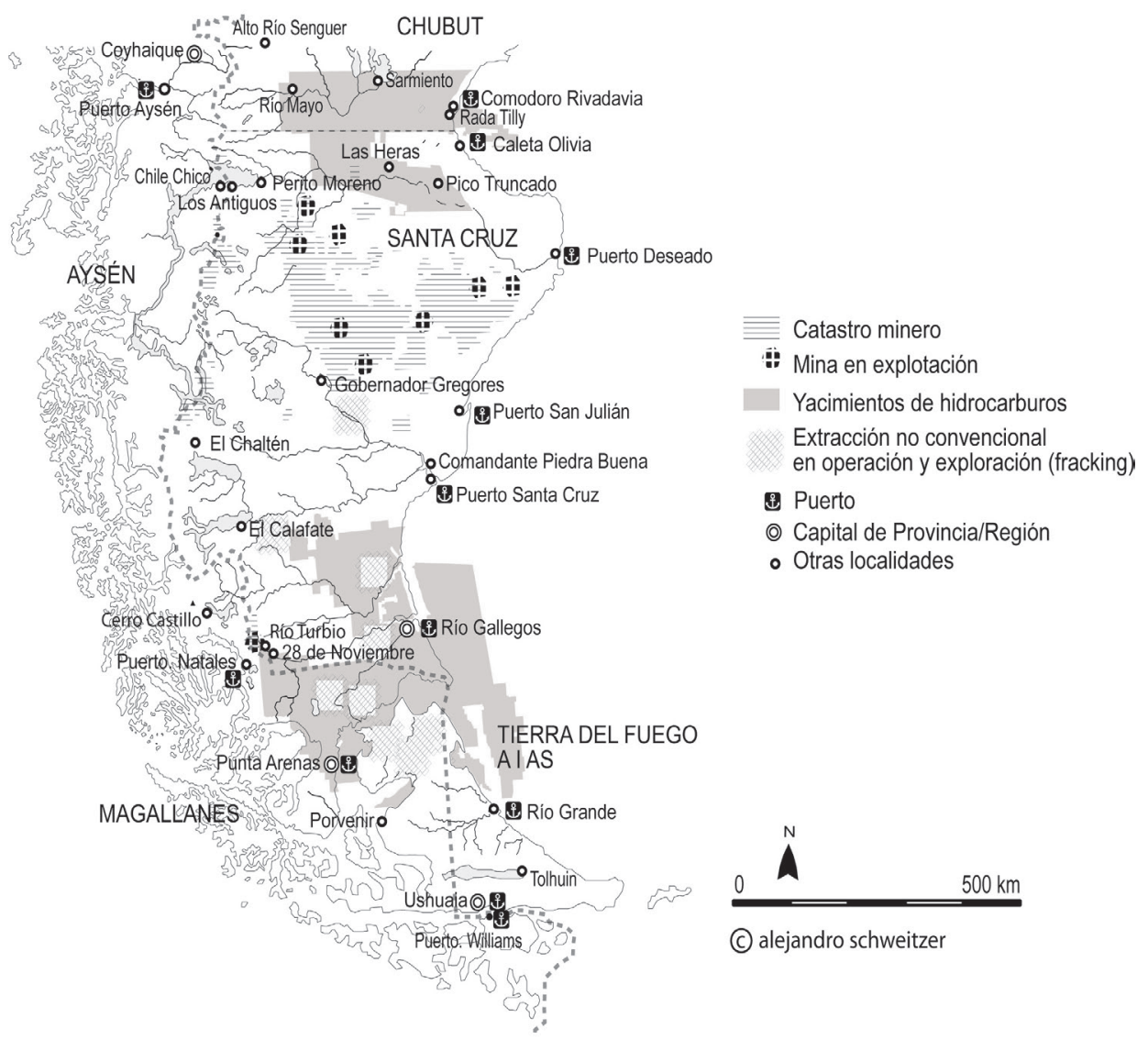

Elaboración: Schweitzer, A. 2019.

\section{El régimen extractivista y sus consecuencias en Santa Cruz}

En la actualidad, en la Provincia de Santa Cruz se encuentran una serie de grandes proyectos de infraestructura en diverso grado de avance con la finalidad de garantizar la permanencia de la situación de frontera de mercantilización y en particular en torno a la minería metalífera en tanto la actividad más dinámica, que representa el principal producto de exportación. Las principales fuentes de estos capitales se encuentran invertidos en particular en los megaproyectos de es el financiamiento chino.

Este financiamiento llega en el marco de tratados de cooperación en materia de infraestructura energética y de transportes negociados y firmados durante los gobiernos de Cristina Fernández y Mauricio Macri. Estos proyectos comprenden la construcción de las represas de Cóndor Cliff y La Barrancosa sobre el río Santa Cruz, obras que se encuentran en estado avanzado en su construcción y una central nuclear con localización todavía no definida, pero que cuenta con el municipio de Caleta Olivia como una de sus alternativas. Los estados provinciales por su parte, favore- 
cidos por las regalías y la coparticipación de impuestos nacionales en el marco de la expansión económica en los años 1990 y 2000, realizan sus propias inversiones para apoyar el mantenimiento de los bajos costos de acceso a energía, suelo e infraestructura vial y portuaria.

Luego de casi un siglo de presencia de la extracción de petróleo y gas, de setenta años de carbón y dos décadas de minería metalífera, se observa en la región la no resolución de la disputa por la renta entre estado y empresas y la completa dependencia de la explotación de los recursos del subsuelo.

Por su parte, la situación política, social, económica y ambiental en las ciudades de la provincia no expresa los beneficios anunciados. Al contrario, se acentúan cada vez con mayor intensidad las contradicciones entre grandes capitales transnacionales y las poblaciones, que se expresan en conflictos por el acceso a condiciones de producción del capital por otras actividades menos dinámicas y asentadas en el mercado interno y condiciones de reproducción de la población, lo que lleva a agudas afectaciones ambientales y la depredación de la naturaleza.

En el plano económico, el perfil rentista bloquea el desarrollo de cualquier otra actividad. Frente a la situación persistente de frontera de mercantilización de la naturaleza, la situación de estos espacios presenta todas las características de lo que algunos autores denominan "maldición de la abundancia” (ACOSTA, 2009), o "maldesarro- llo" (DUMONT; MOTTIN, 1981; TORTOSA, 2011; SVAMPA; VIALE, 2014).

El despliegue de la minería se expresa no solamente en la contaminación de agua y suelo en los espacios de extracción sino también en los efectos de estos impactos en espacios urbanos. Asimismo se expresa en presiones por acceso al suelo urbano, con avance de urbanización sobre espacios de chacras $^{7}$ reduciendo capacidades de producción local de alimentos, por orientación de infraestructuras energéticas priorizando el abastecimiento de establecimientos mineros y la extensión de la electrificación para las empresas, entre otros.

Entre los conflictos sociales derivados del modelo extractivo deben mencionarse al menos los siguientes:

- Inflación local en espacios urbanos. Esta situación se desata como producto de la desigualdad de condiciones laborales y de ingresos entre sectores dependientes del empleo público o de la asistencia estatal, pero también con pequeños comerciantes, cuando al implantarse una actividad extractiva se generan problemas de acceso locales a productos de la canasta básica. Esta situación se percibe en particular en Puerto San Julián, Gobernador Gregores y Perito Moreno, tres localidades cercanas a espacios mineros.

- Especulación inmobiliaria en espacios rurales y urbanos, con avance de la urbanización sobre espacios de chacra, alza de precios de alquileres y problemas de acceso a la vivienda y al suelo urbano. Ante el anuncio de la localización de una actividad como la minería,

7. Las chacras son pequeñas explotaciones de horticultura, fruticultura y ganado de corral que en la $\mathrm{Pa}$ tagonia se implantan en las periferias de las ciudades. 
las expectativas de los pobladores incluyen la construcción de habitaciones y departamentos esperando lograr mayores ingresos por el alquiler. Ocurre algo similar con la ampliación o abertura de comercios. Esta situación se percibe en las tres localidades mencionadas en el punto anterior.

- La localización de las actividades mineras se sitúa por lo general aguas abajo de las ciudades, hacia el este. Estos son los casos de los yacimientos del oeste del Macizo del Deseado en relación a Los Antiguos, Perito Moreno o Gobernador Gregores. Esta situación hace que no existan impactos de las mineras en términos ambientales, con excepción de la ausencia de precauciones en el almacenamiento de productos químicos, como ya ocurrió en Perito Moreno, por parte de la empresa Alex Stewart, contratista de Minera Santa Cruz en 2014 (PERIÓDICO EL EXTREMO SUR. 2014).

- En cambio, en localidades cercanas a los yacimientos de hidrocarburos, como Las Heras, Pico Truncado o Caleta Olivia se verifican altos niveles de contaminación de suelo, napas y aire, lo que afecta la salud de sus pobladores $\mathrm{y}$ la disponibilidad de agua potable en calidad y cantidad.

- Clientelismo del estado provincial y los gobiernos locales respecto a los aportes de las grandes empresas. Los municipios de Gobernador Gregores, Puerto San Julián, Perito Moreno y Puerto Deseado, entre otros, son dependientes de los aportes económicos de las empresas mineras, que operan mediante las agencias de desarrollo, para sostener gastos corrientes y en algunos casos acceder a la compra de equipamientos de salud y seguridad. En algunos casos se ven obligados a aceptar imposiciones que afectan el espacio público, como la cesión de terrenos del estado para la implantación de instalaciones de las empresas. Estas relaciones clientelares colocan al Estado del lado de los grandes capitales transnacionales.
- Cooptación de dirigentes sindicales barriales y de pequeños empresarios, bajo la promesa de trabajo y necesidad de defender fuentes laborales, en particular de la obra pública, contribuyen a que éstos terminan siendo funcionales a los gobiernos de turno.

- Extensión de las redes de trata y prostitución, relacionados con el régimen laboral en explotaciones mineras y de hidrocarburos, donde por el régimen laboral los trabajadores viven aislados de sus familias. La Provincia de Santa Cruz es considerada una de las "receptoras" de este tipo de tráfico, que se asocia asimismo con crecientes casos de violencia de género y feminicidios (KLIPPHAN, 2018)

No todas estas situaciones son motivo del surgimiento de resistencias, esto depende tanto de la intensidad de las afectaciones como de las particularidades de las sociedades locales. En un libro compilado por Svampa y Antonelli (2009) se destacan dos artículos centrados en el surgimiento de las resistencias a la expansión de la megaminería metalífera en la Patagonia. De acuerdo a sus autores, éstas tuvieron origen en el “efecto Esquel”, referido a la localidad de la Provincia del Chubut en la cual triunfó por primera vez la movilización en contra de la minería (MARIN, 2009). En la Provincia del Chubut se formó a fines de la década de 1990 el Movimiento Antinuclear del Chubut, con motivo de resistir la instalación de un basurero nuclear en la localidad de Gastre. Este mismo movimiento sentó las bases de las movilizaciones posteriores, y, en 2002 de la conformación de la asamblea No a la mina y el triunfo contundente del No a la mina en 2003 (SVAMPA; SOLÁ ÁLVAREZ; BOTTARO, 2009; MARIN, 2009). Estas autoras señalan que entre 2002 y 2005 se conformó la Red de Comunidades Afectadas por la Minería, primer antecedente de la Unión 
de Asambleas Ciudadanas, fundada en 2006.

A partir de estas experiencias y ante el anuncio del inicio de obras para los primeros emprendimientos mineros en el Noroeste de la Provincia de Santa Cruz, se conformaron grupos de vecinos, que dieron lugar a las primeras asambleas en las localidades de Perito Moreno, Gobernador Gregores y Los Antiguos, poco después en Las Heras, San Julián y Río Gallegos, preocupados por los primeros accidentes de operación en Cerro Vanguardia. Desde 2009 se realizan reuniones entre localidades vecinas, con participación de asambleas y colectivos de diferentes localidades, unificadas por la resistencia a la contaminación ambiental y la defensa del acceso al agua, suelo y aire puros y de condiciones de reproducción social.

En el espacio nacional entre fines de 2011 y los primeros meses de 2012 diversos hechos complican el escenario, con la aprobación de la Ley Antiterrorista, la agudización de la resistencia en el área de Famatina, en el Noroeste del país o la conformación de la Organización Federal Minera, propuesta desde el gobierno nacional $\mathrm{y}$ asociando a las provincias extractivas, sumado a las primeras represiones de acciones organizadas por estos movimientos.

En un trabajo de 2012 de Schweitzer y Godoy, se plantearon esquemáticamente tres estrategias alternativas empleadas por las empresas, en connivencia con el Estado, dependiendo de las situaciones particulares de cada provincia, región o localidad (SCHWEITZER; GODOY, 2010):

\footnotetext{
a) Implantación de la actividad minera con escasa oposición o directamente con apoyo de la población. En estos casos las acciones de las empresas se reducen al mantenimiento de la imagen positiva, como sucede en Puerto San Julián.
}

b) Implantación de la actividad con oposición moderada. En estos casos las estrategias empresariales se centran en generar consensos en torno a la actividad, proponiendo como eje los beneficios presuntos de la minería hacia el conjunto de la sociedad, y especialmente en los retornos económicos directos para las ciudades involucradas en las zonas de explotación. Éste sería el nivel de actuación en localidades como Gobernador Gregores, Puerto Deseado, Las Heras y otras ciudades donde en su momento existían asambleas que luego resultaron desactivadas y también coinciden con las localizaciones de las agencias de desarrollo.

c) Intento de implantación de la actividad en escenarios de resistencia y oposición a la presencia y actividades de las empresas. En estos casos las estrategias empresariales consisten en una agudización y refuerzo de las medidas para implantar la actividad presionando a los distintos actores. Las acciones promovidas pueden incluir el ejercicio de la amenaza directa o indirecta, la represión incluyendo el uso de la fuerza por parte de organismos de seguridad de las empresas y la participación directa del Estado. Estas medidas hasta ahora sólo se han aplicado en forma muy puntual en Santa Cruz, pero en otras provincias alcanzaron niveles dramáticos, como en el caso particular de la localidad de Andalgalá, Provincia de Catamarca.

\section{Reflexiones finales}

A lo largo del artículo se analizaron las maneras en que la minería metalífera y energética se implantaron en la Patagonia sur, desde hace ya más de un siglo, verificándose la hipótesis planteada de que las mismas dependen y son parte de procesos globales de expansión de las fronteras de mercantilización de la naturaleza. Se analizaron también la evolución y estado actual 
de la actividad minera, en particular y ante la prohibición de la realización de la minería metalífera en dos de las tres provincias de la región, el estudio se centró en la Provincia de Santa Cruz. Se analizó también el estado actual de la expansión de las actividades, los conflictos desatados por los efectos que la misma provocó y cuáles fueron las respuestas por parte de las empresas.

El proceso de expansión de las fronteras de la naturaleza barata (MOORE, 2016; 2017) y de expansión geográfica por transferencia de excedentes (HARVEY, 2004) en la Patagonia Sur fueron posibles por un proceso inicial de genocidio contra pueblos originarios, por el escaso poblamiento posterior y por la concentración de la población en los espacios urbanos. Esto permite plantear una primera pregunta respecto al rol de los propietarios de tierras rurales en el mantenimiento y reproducción del vacío demográfico en gran parte del espacio patagónico, tanto en el periodo de expansión del circuito textil entre 1880 y 1907 y también en la actualidad.

Tanto la expansión de las fronteras de la naturaleza barata, o de mercantilización, como se trata en este artículo, fueron y siguen siendo parte de los procesos de producción del espacio. Al igual que los ajustes espaciales, son fenómenos que ocurren de manera permanente, ante cada despliegue de una nueva actividad. En estos ajustes espaciales se generan contradicciones no solamente entre capitalistas y los trabajadores. Se produce también una segunda contradicción entre el capital y la naturaleza, o, en otras palabras, entre condiciones de producción del capital, en este caso la expansión de la minería, y las condiciones de reproducción de la población, como ocurre con el agua, la energía y algunas infraestructuras que son tanto me- dios de subsistencia de la población como instrumentos de trabajo de las diversas actividades (0'CONNOR, 2001, p. 178), entre otras, de la minería en sus dos modalidades.

En tercer lugar se analizó cómo el estado nacional y las provincias apuntan no solo a garantizar el bienestar general de la población, de acuerdo a principios de derecho, sino que lo hacen principalmente, para asegurar el acceso a alimentos, energía y naterias primas, así como al trabajo barato. Esto se manifiesta en particular en la disputa por las condiciones generales del proceso de producción, que incluyen equipamientos, infraestructuras de transporte, comunicaciones y otras construcciones necesarias para el desarrollo de la actividad minera y la provisión de servicios necesarios para la realización del proceso de producción, entendidas como condiciones técnicas, condiciones sociales y condiciones de reproducción (0'CONNOR, 2001, 2002).

El funcionamiento de las fronteras de la minería metalífera en Santa Cruz y de hidrocarburos en la Patagonia Sur adquieren características de una especie de capitalismo de frontera, o como Rosa Luxemburgo denominaba, como "territorio de acumulación” (LUXEMBURG, 1957), de donde los grandes capitales extraen recursos destinados a asegurar el mantenimiento de sus tasas de ganancia, destruyendo a su paso a la naturaleza humana y extra-humana.

En la actualidad las asambleas ambientales y las resistencias contra la minería en la Patagonia Sur están muy poco activas, con acciones aisladas y con escasa participación en instancias de coordinación nacional o regional. Las presiones sobre los salarios de los empleados públicos y privados, la conflictividad sindical y social, por momentos latente, por momentos explosiva, quitan relevancia a los reclamos por 
mejoras en las condiciones de reproducción de la población, con problemas ambientales que pasan a segundo plano. Existe un desencuentro evidente en la discusión y articulación de las resistencias y las luchas entre, por un lado, mejores condiciones de trabajo y salarios, en el marco global definido por la ley de valor y, por el otro, las formas particulares de reproducción social, es decir, entre las condiciones de producción del capital y condiciones de reproducción social. Los espacios de estas dos esferas son artificialmente diferenciados por múltiples dispositivos, constituyéndose unos como territorios de producción y los otros como territorios de reproducción, pero en ambos casos domina la visión colonial de la naturaleza abstracta, separada de la humanidad, que nos aleja de la lucha por el territorio como construcción social, colectiva y espacio de la vida. Dicho de otro modo, separar en el análisis las luchas por el ambiente respecto a las que a veces los mismos grupos sociales dan por recuperar espacios públicos, por la vivienda, por el salario y la igualdad de género y más en general de la disputa política, termina limitando el desarrollo de los potenciales explicativos de procesos globales y las posibilidades de intervención.

Uno de los desafíos más importantes para los movimientos sociales en resistencia, en defensa del territorio, es lograr superar esta separación artificial de las esferas de la vida y unificar las luchas en amplios movimientos populares que apunten a garantizar las condiciones de reproducción de la naturaleza humana y extra-humana.

\section{Referencias}

ACOSTA, A. La maldición de la abundancia. Quito: Abya-Yala. 2009.
AGOSTO, P (compiladora) Patagonia: resistencias populares a la recolonización del continente. Rosario: América libre. 2008

BASUALDO, F. Evolución y características estructurales de la minería metalífera en la Argentina reciente. La expansión de la actividad y el capital extranjero. IN BASUALDO, F; BARRERA, M; BASUALDO, E. Las producciones primarias en la Argentina reciente. Mineria, petróleo y agro pampeano. Buenos Aires: Centro Cultural de la Cooperación, 2013. pp 9-96.

BRUZZONE, E. Minería argentina. La encrucijada. Buenos Aires: Biblos. 2012.

Diario El nuevo día, 14/06/2013 Legisladores aprobaron presupuesto y canon minero. https://www.eldiarionuevodia.com.ar/local/politica/2013/6/14/legisladoresaprobaron-presupuesto-canon-minero-4299.html

Digesto Jurídico Chubut Ley XVII-No 68 (Antes Ley 5001) de prohibición de la actividad minera metalifera en el ámbito de la Provincia del Chubut http://www.legischubutgov. $\mathrm{ar} / \mathrm{hl} /$ digesto/lxl/XVII-68.html consultado en julio de 2019.

DUMONT, R.; MOTTIN, M. F.; Le maldéveloppment en Amérique Latine. Paris: Seuil, 1981.

El Extremo sur. En Perito Moreno denuncian a minera por evasión y contaminación. 14/04/2014. https://www. elextremosur.com/nota/en-perito-moreno-denuncian-aminera-por-evasion-y-contaminacion/

Energy Information Administration (EIA)-Advances Resources: Technically Recoverable Shale Oil and Shale Gas Resources: Argentina. Washington DC: EIA, 2015,

El observador central "vuelve la "moda" de las agencias de desarrollo local en santa cruz", 2014. http://www. observadorcentral.com.ar/especializadas/desarrollo-local/ vuelve-la-moda-de-las-agencias-de-desarrollo-local-ensanta-cruz/

GIARRACCA, N; TEUBAL, M. Procesos rentísticos y el extractivismo en América latina. IN GIARRACCA, N; TEUBAL, M (coord). Actividades extractivas en expansión ¿reprimarización de la economía argentina?. Buenos Aires: Antropofagia, 2013. pp 45-72.

GODOY, P; FASIOLI, E; VALIENTE, S; SCHWEITZER, A. Valorización de la naturaleza y disputas por el territorio 
en la Provincia de Santa Cruz. Revista de Estudios Regionales y Mercado de Trabajo, №9, 2013, pp 201-216.

HARVEY, D, El nuevo imperialismo. Madrid: Akal, 2004,

INDEC: Censo Nacional de la Actividad Minera (CENAM17). Resultados estadísticos 2016. Buenos Aires: INDEC 2017.

INDEC. Origen provincial de las exportaciones Año 2017. Informes Técnicos vol. 2 n 40 Comercio exterior vol. 2 no 5 . Buenos Aires, 2018.

INFOLEG, Ley 1919 de 1886 del Código de Minería y Ley 24498/95 modificatoria. http://servicios.infoleg.gob.ar/ infolegInternet/anexos/40000-44999/43797/texact.htm\# 1 consultado en septiembre de 2019.

INFOLEG, Ley 14408/55, De provincialización de Territorios Nacionales. http://servicios.infoleg.gob.ar/infolegInternet/verNorma.do\%3Bjsessionid=3A9F96B5889A9251 6602664CFEA70F8E?id=197786 Consultado en julio de 2018.

INFOLEG. Ley 25243/00, Tratado sobre Integración y Complementación Minera, el Protocolo Complementario del Tratado de Integración y Complementación Minera http://servicios.infoleg.gob.ar/infolegInternet/ anexos/60000-64999/62611/norma.htm. Consultado en septiembre de 2019.

INFOLEG, Ley 25675/02 Ley General del Ambiente. http://servicios.infoleg.gob.ar/infolegInternet/anexos/75000-79999/79980/norma.htm Consultado en julio de 2018.

INFOLEG, Ley 26.639/10 Régimen de Presupuestos Mínimos para la Preservación de los Glaciares y del Ambiente Periglacial http:// servicios.infoleg.gob.ar/infolegInternet/anexos/75000-79999/79980/norma.htm Consultado en julio de 2018 .

KLIPPHAN A, Cuáles son las rutas de la trata de personas en la Argentina y qué se hace para combatirlas, Diario Infobae, 1 de septiembre de 2018. https://www.infobae.com/sociedad/2018/09/01/ cuales-son-las-rutas-de-la-trata-de-personas-enla-argentina/

LEGISTDF. Ley 853/11, Actividad Minera: Aplicación de los principios ambientales, establecidos por Ley Nacional № 25.675 y Ley Provincial № 55. http://www.legistdf.gov.ar/lp/leyes/Provinciales/ LEYP853.pdf consultado en septiembre de 2019. MACHADO ARAOZ, H. et al. (Colectivo Voces de Alerta). 15 mitos y realidades de la minería transnacional en la Argentina. Guía para desmontar el imaginario prominero. Buenos Aires: El Colectivo, 2011.

MARÍN, M. El "no a la mina" de Esquel como acontecimiento: otro mundo posible. IN SVAMPA M; ANTONELLI, M. (editoras) Minería transnacional, narrativas del desarrollo y resistencias sociales. Buenos Aires: Biblos, 2009. pp181-204.

Ministerio de Producción de la Provincia de Santa Cruz. Ley 3105/09 de creación del Area de Interés Especial Minero para la realización de actividades mineras http://minpro.gob.ar/legislacion/mineria/leyes/ley-3105.pdf consultado en octubre de 2018.

MO0RE, J. El Auge de la Ecologia-Mundo Capitalista, I: Las fronteras mercantiles en el auge y decadencia de la apropiación máxima, IN Laberinto, 38, 9-26. 2013a.

MO0RE, J. El Auge de la Ecologia-Mundo Capitalista, II: Las fronteras mercantiles en el auge y decadencia de la apropiación máxima. Laberinto, 39, 6-13. 2013b.

MO0RE, J. Crisis: ¿ecológica o ecológico-mundial? Laberinto, 47, 71-75, 2016.

M00RE, J. El fin de la naturaleza barata, o cómo aprendí a dejar de preocuparme por "el" medioambiente y amar la crisis del capitalismo. Relaciones Internacionales Número 33, octubre 2016 - enero 2017. pp 143-174.

0'CONNOR, J. Causas naturales. Ensayos de marxismo ecológico. México: Siglo XXI, 2001. 0'CONNOR, J. ¿Es posible el capitalismo sostenible?. IN ALIMONDA, H. (compilador). Ecología política. Naturaleza, sociedad y utopía. Buenos Aires: CLACSO, 2002.

PENGUE, W. La apropiación y el saqueo de la naturaleza. Conflictos ecológicos distributivos 
en la Argentina del bicentenario. Buenos Aires: Gepama-Heinrich Böll-Lugar ed, 2008.

RODRIGUEZ PARDO, J. Vienen por el oro, vienen por todo. Las invasiones mineras 500 años después. Buenos Aires, CICCUS, 2009.

SCHWEITZER, A Aménagement du Territoire et Intégration Régionale dans le MERCOSUR. Frontières, Réseaux et Dynamiques Transfrontalières, París/Lille: ANRT (microfilm), 2000.

SCHWEITZER, A; GODOY, P, Recursos naturales, ambiente y desarrollo sustentable en la Patagonia austral. Los derechos humanos vistos desde las necesidades, VII Jornadas de Historia Regional, UNPA, Río Gallegos, 4-6 de noviembre de 2010.

SCHWEITZER, A: Fronteras, recursos naturales y crisis en La Patagonia Sur. IN SANDOVAL, JM; ÁLVAREZ, R; SAAVEDRA L. A. (coord.) Integración Geoestratégica, Seguridad, Fronteras y Migración en América Latina. Quito: INREDH, 2011, pp. 33-68.

SCHWEITZER, A; GODOY, P; RAUQUE, M. Recursos naturales y modelos de desarrollo en conflicto en el espacio andino santacruceño. Párrafos Geográficos. Vol. 10:2, 2011, pp. 191-214.

SCHWEITZER A: Desarrollando el desierto. Procesos de frontera en la provincia de Santa Cruz. Revista de Ciencias Sociales año 5 №24, 2013, pp 51-70.

SCHWEITZER, A; GODOY, P; FASIOLI, E, Crisis, valorización de la naturaleza y territorios en la Provincia de Santa Cruz. IN FURLONG, A; NETZAHUALCOYOTZI, R; SANDOVAL Juan Manuel (coord.) Integración en el Continente Americano. Planes geoestratégicos, seguridad regional y fronteriza. Puebla: Benemerita Universidad de Puebla, 2015, pp 162-187.

SCHWEITZER, A. La Patagonia sur como espacio global para la expansión del capital transnacional. Revista Theomai, Estudios críticos sobre sociedad y desarrollo, $n^{\circ} 34$ segundo semestre. 2016, Pp139-151.

SCHWEITZER, A; VALIENTE, S. Extractivismo, megaminería, e hidrocarburos en la Patagonia sur
Argentina, IN RAMÍREZ, M; SCHMALZ, S. ¿Fin de la bonanza?. Entradas, salidos y encrucijadas del extractivismo. Buenos Aires: Biblos. 2018.

Secretaria de Estado de Minería. Plano de proyectos mineros 2016. Ministerio de Producción de Santa Cruz.

SERNAGEOMIN: http://www.sernageomin.cl/mineria/ consultado el 10 de diciembre de 2018.

SVAMPA M; ANTONELLI, M. (editoras). Minería transnacional, narrativas del desarrollo y resistencias sociales. Buenos Aires: Biblos, 2009.

SVAMPA, M; SOLÁ ÁLVAREZ, M; BOTTARO, L. Los movimientos contra la minería metalífera a cielo abierto: escenarios y conflictos. Entre el "efecto Esquel" y el "efecto La Alumbrera". IN SVAMPA M; ANTONELLI, M. (editoras). Minería transnacional, narrativas del desarrollo y resistencias sociales. Buenos Aires: Biblos, 2009. pp. 123-180.

SVAMPA, M., SLIPAK, A. China en América Latina: Del Consenso de los Commodities al Consenso de Beijing. Revista Ensambles primavera 2015, año 2, n.3, pp. 34-63.

SVAMPA, M; VIALE, E. Maldesarrollo. La Argentina del extractivismo y el despojo. Buenos Aires: Katz, 2014.

TAPIA; M: Cuando el Capital es el que manda: la lógica extractiva y la funcionalidad del Estado. XVI Congreso Internacional sobre Integración regional, fronteras y globalización en el continente americano. Medellín. Octubre de 2017. En prensa.

TORTOSA, J.M. Maldesarrollo y mal vivir. Pobreza y violencia a escala mundial. Quito: Abya Yala, 2011.

VELÁZQUEZ, G. et al. Calidad de vida en Argentina. Ranking del bienestar por departamentos (2010). Tandil: Universidad Nacional del Centro de la Provincia de Buenos Aires, 2014.

World Gold Council - Gold Demand Trends Third Quarter 2018|November 2018. https://www.gold. org/ consultado el 14 de enero 2019. 
RESUMEN

La Patagonia sur es desde la década de 1990 territorio de expansión de la minería por parte de grandes capitales transnacionales. Con yacimientos a ambos lados de la cordillera de los Andes, se trata de una actividad inscrita en dinámicas globales de avance de las fronteras de mercantilización de la naturaleza, potenciada por reformas neoliberales. Luego de más de veinte años, los dispositivos que deberían apuntar a aprovechar la renta minera para mejorar las condiciones de reproducción de la población, muestran que estos objetivos no solo no se cumplieron sino que redundaron en empeoramiento de esta situación. En este trabajo se expone un marco para interpretar estos procesos, en un segundo momento se describe el estado actual de la minería en la Patagonia sur y en particular en la Provincia de Santa Cruz, y en tercer lugar algunas de las consecuencias sociales de los problemas ambientales provocados por la actividad así como el estado actual de las resistencias. Al finalizar se aportan algunas reflexiones a modo de conclusión.

\section{PALABRAS-CLAVE}

producción del espacio. Fronteras. Mercantilización de la naturaleza. Minería metalífera. Hidrocarburos. Patagonia sur.

\section{ABSTRACT}

Since the 1990s, southern Patagonia has been a territory for the expansion of mining by large transnational capitals. With deposits on both sides of the Andes mountain range, it is an activity inscribed in global dynamics of advancing the frontiers of the commodification of nature, promoted by neoliberal reforms. After more than twenty years, the devices that should aim to take advantage of the mining income to improve the reproduction conditions of the population, show that these objectives were not only not fulfilled, but also led to a worsening of this situation. In this work a framework is presented to interpret these processes, in a second moment the current state of mining in Patagonia and in particular in the Province of Santa Cruz is described and in third place some of the social consequences of the environmental problems provoked by the activity as well as the current state of the resistances. When finalizing some reflections are contributed by way of conclusion.

\section{KEYWORDS}

Production of space. Borders. Commodification of nature. Metalliferous mining, hydrocarbons. Southern Patagonia.

Recebido em: 22/01/2019

Aprovado em: 25/11/2019 